ARTÍCULOS ORIGINALES

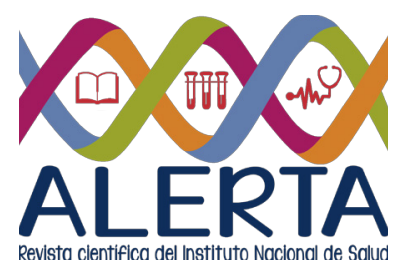

Revista clentifica del instituto Nacional de Saluc

\title{
Evaluación de la indicación de cateterismo cardíaco diagnóstico a pacientes con enfermedad arterial coronaria
}

\author{
Neftalí Alexander Granados Flores ${ }^{1}$
}

${ }^{1}$ Instituto Salvadoreño del Seguro Social

\section{Recibido: 2 de marzo de 2018 Aceptado: 20 de marzo de 2018} Correspondencia: alexander_graflo@hotmail.com

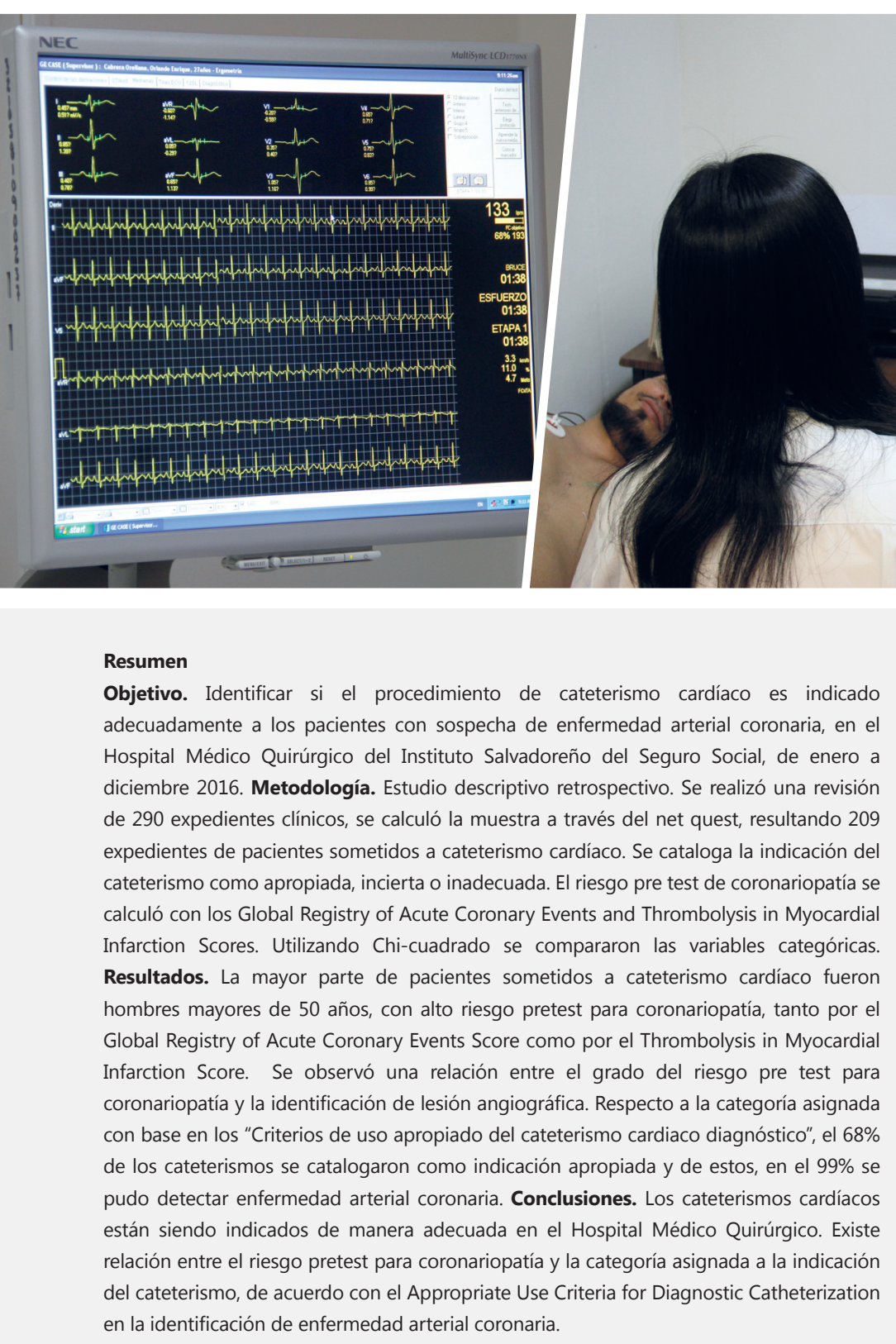

Palabras claves: cateterismo cardíaco, Global Registry of Acute Coronary Events Score, Thrombolysis in Myocardial Infarction Score, enfermedad arterial coronaria.

\section{Abstract}

Objective. To identify if cardiac catheterization is indicated properly to patients suspected of coronary artery disease in Hospital Medico Quirúrgico (a third level establishment pertaining to Instituto Salvadoreño del Seguro Social), from January to December 2016

Methodology. Descriptive retrospective study. Patient's clinical records were reviewed, categorizing the procedure's indication as: appropriate, uncertain or inadequate. The pre-procedure risk score was calculated using the Global Registry of Acute Coronary Events and Thrombolysis in Myocardial Infarction Scores. Chi square test was used to compare categorical variables. Results. Most of the patients that underwent cardiac catherization were male, aged 50 years or more, with a high pre-procedure risk score for coronary disease, calculated by the Global Registry of Acute Coronary Events and Thrombolysis in Myocardial Infarction Scores. A relationship was observed between the pre-procedure risk score and the presence of angiographic injury. Regarding the category assigned, according to the Appropriate Use Criteria for Diagnostic Catheterization, 68\% of the catheterizations were classified as appropriate, detecting coronary artery disease in $99 \%$ of them. Conclusions. Cardiac catherization is being indicated properly at Hospital Medico Quirúrgico. There is a relationship between the preprocedure risk score and the category assigned to the catherization, according to the Appropriate Use Criteria for Diagnostic Catheterization in the diagnosis of coronary artery disease.

Keywords: cardiac catheterization, Global Registry of Acute Coronary Events Score, Thrombolysis in Myocardial Infarction Score, coronary artery disease. 


\section{Introducción}

La enfermedad arterial coronaria o cardiopatía isquémica es la denominación genérica de un grupo de síndromes íntimamente relacionados que producen isquemia miocárdica. Dentro de ellas se encuentra la arteriosclerosis coronaria, la cual afecta las paredes de las arterias coronarias con gran tendencia a obstruirlas, incapacitando el suministro de oxígeno necesario para una determinada área del músculo cardiaco. Esto dificulta el funcionamiento del mismo y puede ocasionar un infarto agudo al miocardio ${ }^{1}$. El diagnóstico de infarto al miocardio se realiza primordialmente basado en la historia clínica, hallazgos electrocardiográficos y enzimas cardíacas, principalmente troponinas séricas y la fracción MB (procedentes del musculo esquelético y del cerebro) de la creatincinasa y pruebas de imágenes como la ecocardiografía y la coronariografía2, 3 .

Según la Organización Mundial de la Salud (OMS), las enfermedades del corazón y los accidentes cerebrovasculares causan la muerte a más de 17 millones de personas cada año, 5, 6 . Se prevé que para el año 2020 estas enfermedades sean la primera causa de defunción y discapacidad y que el número de víctimas aumente a 20 millones y a 24 millones en el $2030^{7,8,9}$.

Según las estimaciones del Informe Sobre la Salud en el Mundo de la OMS, el 12.6\% (7.2 millones) de todas las muertes en el mundo, ha sido causadas por la cardiopatía isquémica ${ }^{10}$.

En el Instituto Salvadoreño del Seguro Social (ISSS) la cardiopatía isquémica, durante el año 2010, fue la sexta causa de muerte (tasa de mortalidad de 67.6 por 100 mil habitantes). Durante el año 2015, fue la segunda causa de muerte. En mujeres fue la primera causa de muerte y en hombres, la cuarta ${ }^{11,12}$.
El cateterismo cardíaco es, hasta la fecha, el gold standard para el estudio de la permeabilidad de las arterias coronarias. No solo brinda información sobre la luz del vaso, sino que además permite un abordaje terapéutico ${ }^{13,14}$. Esta técnica se basa en la administración por vía intravascular de un contraste radiopaco (es una técnica invasiva). Posteriormente se realiza una prueba de rayos $\mathrm{X}$ para revelar la morfología del árbol arterial, así como sus distintos accidentes vasculares, émbolos, trombosis, aneurismas, estenosis ${ }^{15}$. Esta técnica es recomendable utilizarla en los casos que se requiera definir la presencia o la severidad de una lesión cardíaca sospechada que no se pueda evaluar adecuadamente por técnicas no invasivas ${ }^{16}$.

La angiografía es una prueba diagnóstica que ha sido investigada desde la década de $1980^{17}$. La proporción de angiografías apropiadas ha variado en la literatura: del $49 \%$ pasó al $84 \%$, según la ubicación del estudio, población y método de determinación ${ }^{18,19}$. Los pacientes son seleccionados para realizar este procedimiento con base en el riesgo pre test, definido como la probabilidad de enfermedad arterial coronaria previo a cateterismo cardíaco ${ }^{20,21}$.

El cateterismo cardíaco es practicado en el ISSS desde 1991. En los últimos cuatro años (2014-2018) se han practicado 3566 intervenciones con un promedio anual de $891.5^{22}$. Otros estudios ${ }^{9,22}$ reportan que anualmente consultan en promedio 135 pacientes con infarto al miocardio. En el Hospital Médico Quirúrgico del ISSS, en promedio, se realizan de 15 a 20 procedimientos de cateterismo cardíaco cada mes ${ }^{22}$.

Este estudio pretende identificar si el procedimiento de cateterismo cardíaco es indicado adecuadamente a los pacientes con sospecha de enfermedad arterial coronaria en el Hospital Médico Quirúrgico durante el año 2016. 


\section{Metodología}

Este es un estudio descriptivo retrospectivo que consiste en la revisión de expedientes clínicos de pacientes a quienes se les ha tratado con cateterismo cardíaco diagnóstico, durante el periodo de enero a diciembre 2016.

De un total de 455 expedientes de pacientes sometidos a cateterismos cardíacos, tanto diagnóstico (338) como terapéutico (117), atendidos en el Hospital Médico Quirúrgico del ISSS durante el año 2016, se tomaron solamente los expedientes con cateterismo de tipo diagnóstico. En total fueron seleccionados 338 expedientes. No se tomaron en cuenta los expedientes con información incompleta. Se excluyeron 48 expedientes incompletos. A partir de los expedientes seleccionados, (290) se calculó la muestra a través del Net Quest ${ }^{23}$, con un intervalo de confianza del $98 \%$. Finalmente, la muestra quedó conformada por 209 expedientes de pacientes sometidos a cateterismo cardíaco.

La información fue recolectada a través de una hoja de cotejo aprobada por el comité de ética del ISSS. Los datos recolectados fueron digitados y organizados a través del software Microsoft Office Excel 2003. El análisis de la información se realizó a través del Statistical Analytics Software (SAS) tomando como resultado significativo $p<0.05$. Se realizaron cálculos de frecuencias y prueba de chi cuadrado. La indicación del cateterismo diagnóstico fue clasificada en tres categorías con base en Appropriate Use Criteria for Diagnostic Catheterization $(A \cup C)^{21}$, una guía propuesta en el 2012 que comprende 166 indicaciones desarrolladas y catalogadas por un grupo técnico independiente y separadas en una escala de puntaje del 1 a 9, para designar el uso del cateterismo cardíaco como: apropiado, incierto y no apropiado. Los resultados de esta guía fueron validados a través de un estudio observacional de cohorte multicéntrico realizado en la Red de Atención Cardíaca, en Ontario, Canadá24.
Además, se calculó el riesgo pretest a través del score GRACE (The Global Registry of Acute Coronary Events) y el TIMI (Thrombolysis In Myocardial Infarction). Ambos evalúan el riesgo de muerte después de un evento cardiovascular con base en características del paciente, tanto demográficas, clínicas, electrocardiográficas y de laboratorio. Estas escalas se aplican sumando un punto por cada variable que esté presente según la información del paciente y según su resultado se clasifican los pacientes en tres grupos de riesgo de acuerdo con el riesgo de presentar un desenlace fatal: riesgo muy bajo, riesgo bajo con probabilidad de 4.7 \% - 8.3\%; intermedio, con un riesgo entre $13.2 \%$ y $19.9 \%$; y alto, lo cual indica un riesgo entre $26.2 \%$ y $40.9 \%^{25-27}$. Para el cálculo del puntaje de ambas escalas se utilizó la calculadora MediCalc ${ }^{28}$.

Los datos son presentados a través de tablas de contingencia de doble entrada y gráficas de barras con datos enteros donde se categorizan y correlacionan los datos, según factores demográficos de los sujetos en estudio y las categorías de los cateterismos cardíacos.

\section{Resultados}

Del total de procedimientos realizados en la sala de cateterismo cardíaco, el $44 \%$ corresponde a cateterismos cardíacos; y de estos, el $74 \%$ son de primera vez. De los 209 expedientes revisados, el $66.5 \%$ pertenece a pacientes del sexo masculino. El $81.8 \%$ tiene más de 50 años de edad. Se observa que a través de las líneas de tendencia (gráfico 1) a medida incrementa la edad aumenta el número de cateterismos cardíacos. El 79 \% son cotizantes activos del ISSS y el $84 \%$ proviene de la zona urbana.

Con base en el cálculo del riesgo pretest a través del score TIMI, el $59.8 \%$ de los pacientes tiene un riesgo muy alto. El $92 \%$ de los pacientes tienen enfermedad arterial coronaria (gráfico 2). 
De acuerdo con los criterios AUC, el $67.4 \%$ de los cateterismos cardíacos diagnósticos fueron indicados de forma apropiada. En el 99.2\% de estos se encontró enfermedad arterial coronaria (gráfico 3).

El cruce de las variables de sexo y edad de los pacientes respecto a la probabilidad de enfermedad arterial coronaria, riesgo prestest y la categoría asignada por los criterios AUC respecto a la probabilidad de documentar lesión angiográfica significativa, se presentan en el gráfico 4. Se observa que los pacientes mayores de 50 años presentan mayor riesgo pretest y es en estos pacientes que efectivamente se documenta lesión angiográfica significativa. La prueba de chi cuadrado muestra que la enfermedad arterial coronaria, aunque fue mayormente documentada en hombres, es independiente del sexo $(0.7475, p>0.05)$. Aunque presenta una tendencia al incremento respecto a la edad del paciente, no hay significancia estadística entre estas variables (2.63, p >0.62). Existe relación entre el riesgo pre test $(73.56, p<0.05)$ y la categoría AUC del cateterismo cardíaco diagnóstico con la probabilidad de documentar coronariopatía (159.7, p <0.05).

\section{Discusión}

El $66.5 \%$ de pacientes estudiados corresponde al sexo masculino, que coincide con la literatura internacional, donde este grupo etario se ve más afectado por enfermedad cardiovascular. En las mujeres se produce un pico de casos en el rango de edad entre los 60-69 años. Esto podría explicarse por la finalización de protección estrogénica, que coincide con la menopausia y conlleva a un aumento del riesgo cardiovascular en esta población ${ }^{14}$. De los pacientes sometidos a cateterismo cardíaco, el $82 \%$ son mayores de 50 años, presentando una mayor utilización del cateterismo cardíaco a mayor edad del paciente. El riesgo pretest utilizado para selección de los pacientes, es dependiente de la edad, por lo que se justifica aún más la utilización de este recurso ${ }^{6,25}$.

Al calcular el riesgo pretest de los pacientes, a través del score TIMI y GRACE, la mayoría (59.8\%) presenta un riesgo catalogado como alto. Es decir, la mayoría de sujetos sometidos a cateterismo cardíaco, tienen un riesgo alto de enfermedad arterial coronaria. Esto se relaciona también con la distribución por edades de los sujetos de estudio. En el $92 \%$ de estos cateterismos se pudo demostrar lesión angiográfica significativa. En el caso de los catalogados como de muy bajo riesgo, solo en el $22 \%$ se encontró coronariopatía, por lo que los dos modelos anteriores se usan de manera rutinaria en los servicios de urgencias, para determinar el subgrupo de pacientes con infarto agudo al miocardio de más alto riesgo, a quienes se les debe realizar intervención invasiva.

Del total de cateterismos cardíacos realizados en el laboratorio de hemodinámica del ISSS, en el Hospital Médico Quirúrgico como centro nacional de referencia, dos terceras partes son cateterismos cardíacos de primera vez. Lo anterior deja en claro el uso habitual de este recurso para el diagnóstico de pacientes con sospecha de enfermedad arterial coronaria ${ }^{9,22}$.

Analizando la categoría asignada al cateterismo según los criterios $\mathrm{AUC}^{21}$ y la presencia de enfermedad coronaria por cateterismo, se documentó que el $67.5 \%$ fueron catalogados como una indicación apropiada, $14.3 \%$ inadecuada y $18.2 \%$ como incierto. Es de reconocer la aceptable selección de pacientes en el Hospital Médico Quirúrgico, pues si se compara con los datos obtenidos del estudio de validación ${ }^{24}$ realizados en el 2015 en centros canadienses, que reportan $58.2 \%$ apropiados, $10.8 \%$ inadecuados y $31 \%$ incierto. El 99\% de los cateterismos catalogados como apropiados efectivamente tenían presencia de enfermedad arterial coronaria. Así, la probabilidad de documentar lesión angiográfica por cateterismo guarda relación con la categoría asignada a la indicación del mismo. En el grupo dentro de la categoría incierta, el 28\% de estos pacientes reflejó coronariopatía por cateterismo cardíaco, por lo que debe optimizarse el análisis de estos pacientes. 
Gráfico 1. Distribución de cateterismos por sexo y edad

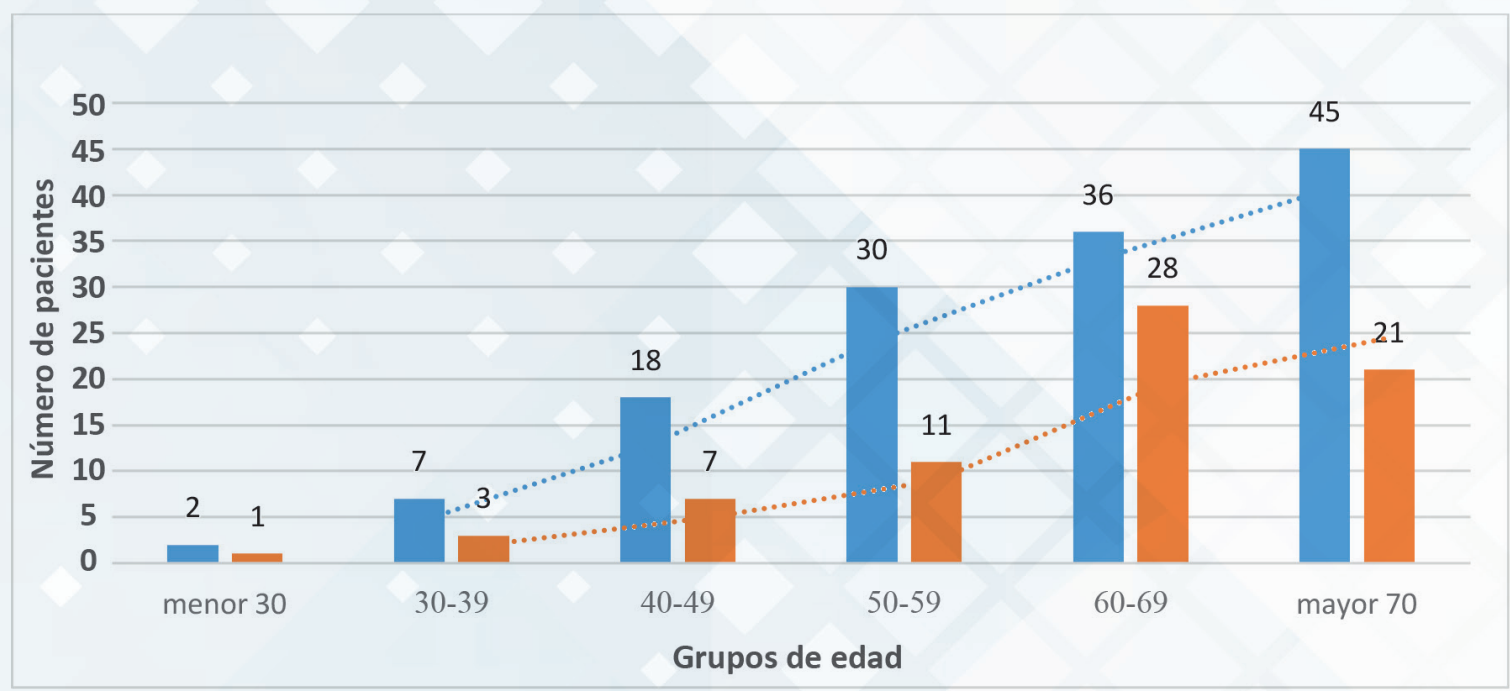

Masculino

- Femenino

Fuente: Elaboración propia a partir de datos generados en el estudio

Gráfico 2. Presencia de enfermedad arterial coronaria según riesgo pre test

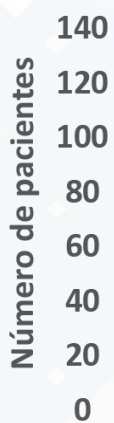

100

80

60

40

20

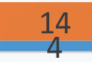

18

Muy Bajo

Bajo

Riesgo pre test

Con enfermedad coronaria $\quad$ Sin enfermedad coronaria

Fuente: Elaboración propia a partir de datos generados en el estudio 
Gráfico 3. Presencia de enfermedad arterial coronaria según categoría (AUC) de la indicación del cateterismo

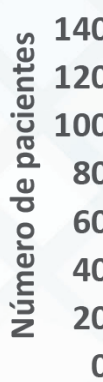

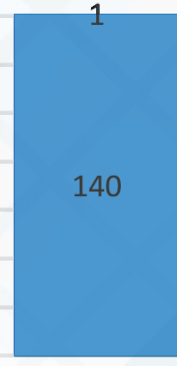

Adecuado

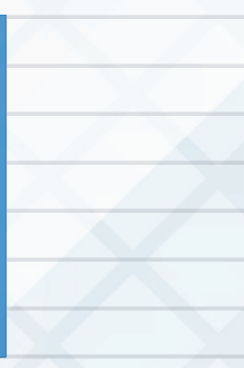

1

Inadecuado

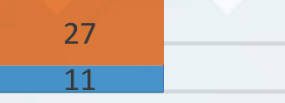

Incierto

\section{Categoria AUC}

$\square$ Con enfermedad coronaria

- Sin enfermedad coronaria

Fuente: Elaboración propia a partir de datos generados en el estudio

Gráfico 4. Cateterismos cardíacos positivos a enfermedad arterial coronaria según edad, sexo y riesgo pre test.

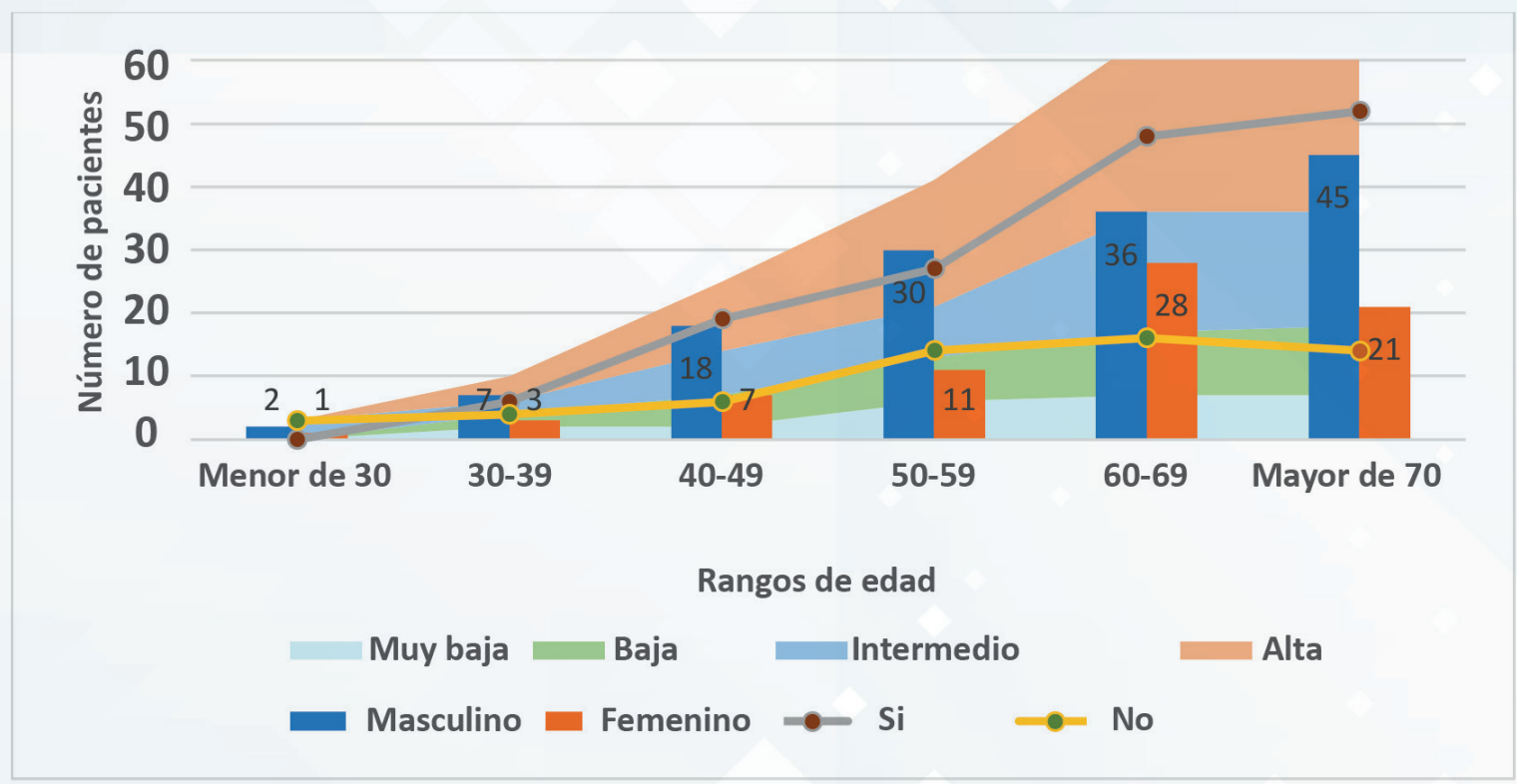

Fuente: Elaboración propia a partir de datos generados en el estudio 
Al analizar las variables edad y sexo del paciente y la probabilidad de documentar enfermedad arterial coronaria, no se encontró significancia estadística. Sí se encontró significancia, entre la presencia de enfermedad arterial coronaria documentada por cateterismo cardíaco y el riesgo pretest, pues este toma otras variables como la historia clínica, hallazgos electrocardiográficos y elevación enzimática para asignar el riesgo del paciente, así como con la categoría asignada por los criterios AUC, pues evalúan el contexto clínico de la indicación del cateterismo cardíaco con base en el riesgo pretest del paciente.

Las limitaciones de este estudio radican en la ausencia de un registro electrónico de los pacientes intervenidos en sala de cateterismo cardíaco. Solo se cuenta con un registro manuscrito de datos escasos del paciente, lo que dificultó la recolección de los expedientes a evaluar. Además, en el estudio se excluyeron aquellos expedientes incompletos (48 en total) y no se clasificó la población dependiendo de su categoría de asegurado (cotizante, beneficiario o pensionado), lo cual pudo haber modificado los resultados demográficos que arrojó el estudio.

Es importante capacitar al personal médico sobre los criterios de uso apropiado del cateterismo cardíaco diagnóstico (AUC) para optimizar la oportuna utilización de este recurso. De esta forma, se puede proporcionar al paciente la mejor opción diagnóstica y terapéutica. Implementar en las diferentes áreas de atención del paciente con sospecha de enfermedad arterial coronaria el cálculo del riesgo pre test mediante el score GRACE y TIMI como herramienta para determinar qué paciente se beneficiaría más de un cateterismo cardíaco diagnóstico. Ampliar el conocimiento entre el personal médico sobre cardiopatía isquémica y las pautas de diagnóstico mundialmente establecidas, para proporcionar al paciente una atención médica de calidad y un manejo adecuado.

\section{Conclusiones}

La mayoría de cateterismos realizados en el Hospital Médico Quirúrgico por sospecha de enfermedad arterial coronaria son indicados adecuadamente.

El riesgo pre test para la enfermedad arterial coronaria de la mayoría de los pacientes sometidos a cateterismo cardíaco es catalogado como alta.

La enfermedad arterial coronaria fue documentada por cateterismo cardíaco en mayor proporción en pacientes masculinos mayores de 50 años.

A mayor riesgo pre test mayor probabilidad de documentar lesión angiográfica por cateterismo cardíaco.

A mejor categoría asignada por los criterios AUC mayor probabilidad de documentar lesión angiográfica por cateterismo cardíaco.

\section{Referencias bibliográficas}

1. Selwyn AP, Braunwald E. Ischemic heart disease. En: Kasper DL, Braunwald E, Fauci AS, Hauser SL, Longo DL, Jameson JL. Harrison's Principles of Internal Medicine. 16 Ed. New York: McGraw-Hill Interamericana; 2005; v. 2: p. $1434-1438$.

2. Little WC, Constantinescu M, Applegate RJ, Kutcher MA, Burrows $\mathrm{MT}$, Kahl FR, et al. Can coronary angiography predict the site of a subsequent myocardial infarction in patients with mild-tomoderate coronary artery disease? Circulation. noviembre de 1988;78(5 Pt 1):1157-66.

3. Mohareb MM, Qiu F, Cantor WJ, Kingsbury KJ, Ko DT, Wijeysundera HC. Validation of the Appropriate Use Criteria for Coronary Angiography: A Cohort Study. [En línea]. Ann Interna Med. 2015 Mar 10. doi: 10.7326/M14-1889 [citado 17 de mayo del 2016].

4. Centro de noticias de la ONU. OMS publica atlas sobre cardiopatías y enfermedades cardiovasculares [Internet]. Noticias ONU. 2004 [citado 27 de mayo de 2018]. Disponible en: https:// news.un.org/es/story/2004/09/1042111

5. OMS. OMS | ¿Cuál es la enfermedad que causa más muertes en el mundo? [Internet]. WHO. [citado 27 de mayo de 2018]. Disponible en: http://www.who.int/features/qa/18/es/ 
6. Colin, Tidy. Epidemiology of Coronary Heart Disease. CHD information. Patient [Internet]. [citado 27 de mayo de 2018]. Disponible en: https://patient.info/doctor/epidemiology-ofcoronary-heart-disease

7. Digestyc. Documentos - DIRECCION GENERAL DE ESTADISTICA Y CENSOS [Internet]. [citado 27 de mayo de 2018]. Disponible en: http://www.digestyc.gob.sv/index.php/temas/des/poblacion-yestadisticas-demograficas/vitales/documentos-vitales.html

8. Monterrosa Linares $\mathrm{H}$. "Estudio descriptivo sobre el perfil de factores de riesgo cardiovascular y estudio de gabinete en los pacientes que se realizaron intervención percutánea coronario en el laboratorio de cateterismo cardíaco del ISSS durante el periodo 2010" [Tesis Doctoral]. Universidad de El Salvador, Facultad de Medicina; 2010.

9. Ruiz Ruiz FJ, Casanovas Lenguas JA, Servicio de Medicina Interna y Cardiología del Hospital Clínico Universitario. Revistas de la Sociedad Aragonesa de Cardiología. Chlamydia y aterosclerosis. [En línea] 2003 [accesado el 10 abril 2017]; 6(6): Disponible en: http://www. cardioaragon.com/doc.php?op $=$ revista3\&id $=54 \&$ id $2=6$

10. Mitchell RN, Cotran RS. Cardiopatía isquémica. En: Robbins, RS, Kumar V, Collins T, Robbins SL. Patología estructural y funcional. 6 ed. Madrid: McGraw- Hill Interamericana: p. 140 - 143.

11. Michel W, Cleman MD. Methods of treatment: coronary angioplasty and interventional cardiology. [CD-ROM]. 2005. New Haven. Yale University; 2004.

12. Baim DGrossman W. Grossman's cardiac catheterization, angiography, and intervention. Philadelphia, PA: Lippincott, Williams \& Wilkins; 2000. Capítulo 2, pág. 34.

13. Cheitlin MD, Sokolow M, McIlroy MB. Clinical Caridiology. 6 ed. Connecticut: Appleton \& Lange; 1993: Pág 84 - 97.

14. Fauci AS, Kasper DL, Braunwald E, Hauser SL, Longo DL, Jameson $J \mathrm{~L}$, et al. Harrison's principles of internal medicine. Vol 1. 17th ed. New York: Mc Graw Hill; 2008. 347- 349 p.

15. Patel MR, Bailey SR, Bonow RO, Chambers CE, Chan PS, Dehmer GJ, et al. ACCF/SCAI/AATS/AHA/ASE/ASNC/HFSA/HRS/SCCM/ SCCT/SCMR/STS 2012 Appropriate Use Criteria for Diagnostic Catheterization. Journal of the American College of Cardiology. mayo de 2012;59(22):1995-2027.

16. Libby P, Bonow RO, Mann DL, Zipes DP. Braunwald's heart disease: a textbook of cardiovascular medicine. 8 ed. Washington: McGrawHill Interamericana; 2008. Pág 47- 49.

17. Michel W, Cleman MD. Methods of treatment: coronary angioplasty and interventional cardiology. [CD-ROM]. 2005. New Haven. Yale University; 2004.
18. McPhee SJ, Papadakis MA, Tierney LM. Current medical diagnosis \& Treatment. Coronary Heart Disease. 46 ed. Florida: McGraw- Hill Interamericana; 2007: p. 345 - 354.

19. Libby P, Bonow RO, Mann DL, Zipes DP. Braunwald's heart disease: a textbook of cardiovascular medicine. 8 ed. Washington: McGrawHill Interamericana; 2008.

20. Anderson RW, Milano CA. Cardiopatía adquirida: insuficiencia coronaria. En: Townsend CM, Beauchamp RD, Mattox KL, Evers BM. Sabinston tratado de patología quirúrgica. 16 ed. México. McGraw-Hill Interamericana; 2003; v. 2: p. 1451 - 1453.

21. ACCF / SCAI / AATS / AHA / ASE / ASNC / HFSA / HRS /SCCM / SCCT / SCMR / STS. 2012 Criterios de uso apropiados para la cateterización diagnóstica. Disponible en: http://content.onlinejacc. org/cgi/content/full/j.jacc.2012.03. 003v1

22. Bonilla, Reyes M. Infarto agudo al miocardio en pacientes con cateterismo cardiaco atendidos en el hospital médico quirúrgico periodo de enero 2002 a diciembre 2004 [Tesis Doctoral]. Universidad de El Salvador. Facultad de Medicina; 2005.

23. Netquest. Calcula qué tamaño de muestra necesitas [Internet]. [citado 27 de mayo de 2018]. Disponible en: https://www.netquest. com

24. Mohareb MM, Qiu F, Cantor WJ, Kingsbury KJ, Ko DT, Wijeysundera $\mathrm{HC}$. Validation of the appropriate use criteria for coronary angiography: a cohort study. Ann Intern Med. 21 de abril de 2015;162(8):549-56.

25. Wynder E. Epidemiological aspects of coronary artery disease. New York: New York Academy of Sciences; 1963; pág 232.

26. Guadalajara Boo JF. Cardiología. México, D.F.: Méndez Editores; 2006. 1194 p.

27. Badimon JJ, Fuster V, Chesebro JH, Badimon L. Coronary atherosclerosis. A multifactorial disease. Circulation. marzo de 1993;87(3 Suppl):II3-16.

28. MediCalc :: Sistema de Calculadora Médica :...: SCYMED :...: [Internet]. [citado 27 de mayo de 2018]. Disponible en: http://www.scymed.com/ es/smnxfd/smnxfdaa.htm

\section{Forma recomendada de citar}

Granados Flores NA. Evaluación de la indicación de cateterismo cardíaco diagnóstico a pacientes con enfermedad arterial coronaria Revista ALERTA. 2018;1(1): 18-25. 